Pacific

Journal of

Mathematics

AN EICHLER-ZAGIER MAP FOR JACOBI FORMS OF HALF-INTEGRAL WEIGHT

M. MANICKAM AND B. RAMAKRISHNAN 


\title{
AN EICHLER-ZAGIER MAP FOR JACOBI FORMS OF HALF-INTEGRAL WEIGHT
}

\author{
M. MANICKAM AND B. RAMAKRISHNAN
}

\begin{abstract}
We construct an Eichler-Zagier map for Jacobi cusp forms of half-integral weight. As an application, we show there exists no Hecke-equivariant map from index 1 to index $p$ ( $p$ prime), when the weight is half-integral.
\end{abstract}

The aim of this paper is to generalize the Eichler-Zagier map for Jacobi forms of half-integral weight, which is formally defined as

$$
\mathscr{E}_{m}: \sum_{\substack{0>D, r \in \mathbb{Z} \\ D \equiv r^{2}(4 m)}} c(D, r) e\left(\frac{r^{2}-D}{4 m} \tau+r z\right) \mapsto \sum_{0>D \in \mathbb{Z}}\left(\sum_{\substack{r(2 m) \\ r^{2} \equiv D(4 m)}} c(D, r)\right) e(|D| \tau) .
$$

We prove that it is a Hecke-equivariant map from Jacobi cusp forms of weight $k+\frac{1}{2}$ on $\Gamma_{0}(4 M)$, index $m$ and character $\chi$ ( $k$ and $\chi$ are even) into a certain subspace of cusp forms of weight $k$ on $\Gamma_{1}\left(16 m^{2} M\right)$. First we derive this assertion for $m=1$ by proving that $\mathscr{L}_{1}$ maps respective Poincaré series. For the general index $m$, we apply certain operator $I_{m}$ (see (2) for the definition) which changes the index $m$ into index 1 and then apply $\mathscr{L}_{1}$ to obtain the required mapping property.

In order to give a Maass relation for each prime $p$ for Siegel modular forms of half-integral weight and degree two, Y. Tanigawa [1986] obtained a Heckeequivariant map from the space of index 1 Jacobi forms of half-integral weight into certain modular forms of integral weight and he constructed the map $V_{p^{2}}$ from the space of Jacobi forms of index 1 into index $p^{2}$. As a natural question, he asked the existence of a connection between Jacobi forms of index 1 and index $p$ ( $p$ is a prime) in the case of half-integral weight. We show that there is no such Hecke-equivariant map as an application of the nature of the map $\mathscr{E}_{m}$.

Notation and background. Throughout this paper, unless otherwise specified, the letters $k, m, M, N$ will stand for natural numbers and $\tau$ for an element of $\mathscr{H}$, the complex upper half-plane.

MSC2000: primary 11F11, 11F50; secondary 11F37.

Keywords: Modular forms, Siegel modular forms, Jacobi forms. 
For a complex number $z$, we write $\sqrt{z}$ for the square root with argument in $(-\pi / 2, \pi / 2]$, and we set $z^{k / 2}=(\sqrt{z})^{k}$ for any $k \in \mathbb{Z}$.

For integers $a, b$, let $\left(\frac{a}{b}\right)$ denote the generalized quadratic residue symbol. Let $S_{k}(N, \psi)$ denote the space of cusp forms of weight $k$ and level $N$ with character $\psi$. We write the Fourier expansion of a modular form $f$ as

$$
f(\tau)=\sum_{n \geq 1} a_{f}(n) e^{2 \pi i n \tau} .
$$

For $z \in \mathbb{C}$ and $c, d \in \mathbb{Z}$, we put $e_{d}^{c}(z)=e^{2 \pi i c z / d}$. We also write $e_{1}^{c}(z)=e^{c}(z)$, $e_{c}^{1}(z)=e_{c}(z)$, and $e_{1}^{1}(z)=e(z)$. The symbol $a \equiv \square(b)$ means that $a$ is a square modulo $b$. For two forms $f$ and $g$ (either in the space of modular forms of integral weight or in the space of Jacobi forms of half-integral weight), $\langle f, g\rangle$ denotes the Petersson inner product of $f$ and $g$. For a Dirichlet character $\psi$ modulo $4 m$, the twisting operator on modular forms of integral weight is given by

$$
R_{\psi}=\frac{1}{W_{\psi}} \sum_{u \bmod 4 m} \bar{\psi}(u)\left(\begin{array}{cc}
4 m & u \\
0 & 4 m
\end{array}\right),
$$

where $W_{\psi}=\sum_{u \bmod (4 m)} \psi(u) e(u / 4 m)$. It follows that $\left\langle f \mid R_{\psi}, g\right\rangle=\left\langle f, g \mid R_{\bar{\psi}}\right\rangle$, where $f, g \in S_{k}\left(\Gamma_{1}(16 m M)\right)$ and

$$
R_{\psi}: \sum_{n \geq 1} a_{f}(n) e(n \tau) \mapsto \sum_{n \geq 1} \psi(n) a_{f}(n) e(n \tau) .
$$

For a natural number $d$, the operators $U(d)$ and $B(d)$ are defined on formal power series by

$$
\begin{aligned}
& U(d): \sum_{n \geq 1} a(n) e(n \tau) \mapsto \sum_{n \geq 1} a(n d) e(n \tau), \\
& B(d): \sum_{n \geq 1} a(n) e(n \tau) \mapsto \sum_{n \geq 1} a(n) e(n d \tau) .
\end{aligned}
$$

For $n \geq 1$, let $P_{n}$ denote the $n$-th Poincaré series in $S_{k}(N, \psi)$ whose $\ell$-th Fourier coefficient is given by

$$
g_{n}(\ell)=\delta(\ell, n)+2 \pi i^{-k}(\ell / n)^{(k-1) / 2} \sum_{c \geq 1, N \mid c} K_{N, \chi}(n, \ell ; c) J_{k-1}\left(\frac{4 \pi \sqrt{n \ell}}{c}\right),
$$

where $\delta(\ell, n)$ is the Kronecker-delta function, $J_{k-1}(x)$ is the Bessel function of order $k-1$ and $K_{N, \chi}(n, \ell ; c)$ is the Kloosterman sum defined by

$$
K_{N, \chi}(n, \ell ; c)=\frac{1}{c} \sum_{\substack{d(c)^{*} \\ d d^{-1} \equiv 1(c)}} \bar{\psi}(d) e_{c}\left(n d^{-1}+\ell d\right) .
$$




\section{A certain space of cusp forms of integral weight}

For $m, M \in \mathbb{N}$, let $\chi \bmod M$ be a Dirichlet character and $\chi_{m}(n)=\left(\frac{m}{n}\right)$ be the quadratic character modulo $m$ or $4 m$ according as $m \equiv 1$ or $m \equiv 3(\bmod 4)$.

Let

$$
\begin{aligned}
S & =\{\ell \in \mathbb{N}: 1 \leq \ell \leq 4 m, \ell \equiv \square(4 m)\}, \\
S^{*} & =\left\{\ell \in S: p^{2} \mid 4 m M \text { implies } p \nmid \ell, \text { with } p \text { prime }\right\} .
\end{aligned}
$$

If $\ell \in S$, define

$$
S_{k}^{\square, \ell}\left(16 m M, \chi \chi_{m}\right):=S_{k}\left(16 m M, \chi \chi_{m}\right) \mid R_{\ell},
$$

where

$$
R_{\ell}: \sum_{n \geq 1} a(n) e(n \tau) \mapsto \sum_{\substack{n \geq 1 \\-n \equiv \bar{\ell}(4 m)}} a(n) e(n \tau)
$$

For $\ell \in S$, let $t=(\ell, 4 m)$. A formal computation shows that

$$
R_{\ell}=U(t) R(\ell) B(t),
$$

with

$$
R(\ell)=\frac{1}{\varphi(4 m / t)} \sum_{\psi \bmod 4 m / t} \bar{\psi}(-\ell / t) R_{\psi},
$$

where $\varphi(n)$ is the Euler totient function. Using the mapping properties of $U(t)$, $R_{\psi}$ and $B(t)$ in the said order, we verify that $S_{k}^{\square, \ell}\left(16 m M, \chi \chi_{m}\right)$ is a subspace of $S_{k}\left(\Gamma_{1}\left(16 m^{2} M\right)\right)$. Finally we define

$$
S_{k}^{\square}\left(16 m M, \chi \chi_{m}\right)=\sum_{\ell \in S} S_{k}^{\square, \ell}\left(16 m M, \chi \chi_{m}\right) .
$$

\section{Jacobi forms of half-integral weight}

For $\alpha=\left(\begin{array}{ll}a & b \\ c & d\end{array}\right) \in \mathrm{SL}_{2}(\mathbb{R})$, let $\tilde{\alpha}=(\alpha, \phi(\tau))$, where $\phi(\tau)$ is a holomorphic function on $\mathscr{H}$ such that $\phi^{2}(\tau)=t(c \tau+d)$, with $t \in\{1,-1\}$. Then the set $G=\left\{\tilde{\alpha}: \alpha \in \mathrm{SL}_{2}(\mathbb{R})\right\}$ is a group with group law

$$
\left(\alpha_{1}, \phi_{1}(\tau)\right)\left(\alpha_{2}, \phi_{2}(\tau)\right)=\left(\alpha_{1} \alpha_{2}, \phi_{1}\left(\alpha_{2} \tau\right) \phi_{2}(\tau)\right) .
$$

If $\alpha \in \Gamma_{0}(4)$, set

$$
j(\alpha, \tau)=\left(\frac{c}{d}\right)\left(\frac{-4}{d}\right)^{-1 / 2}(c \tau+d)^{1 / 2} .
$$

We set $\alpha^{*}=(\alpha, j(\alpha, \tau))$; the association $\alpha \mapsto \alpha^{*}$ is an injective map from $\Gamma_{0}(4)$ into $G$. Let $G^{J}$ be the set of all triplets $[\tilde{\alpha}, X, s], \alpha \in \mathrm{SL}_{2}(\mathbb{R}), X \in \mathbb{R}^{2}, s \in \mathbb{C}$, $|s|=1$. Then $G^{J}$ is a group, with group law given by

$$
\left[\tilde{\alpha}_{1}, X_{1}, s_{1}\right]\left[\tilde{\alpha}_{2}, X_{2}, s_{2}\right]=\left[\tilde{\alpha}_{1} \tilde{\alpha}_{2}, X_{1} \alpha_{2}+X_{2}, s_{1} s_{2} \cdot\left(\operatorname{det}\left(\begin{array}{c}
X_{1} \alpha_{2} \\
X_{2}
\end{array}\right)\right)\right] .
$$


The stroke operator $\left.\right|_{k+1 / 2, m}$ is defined on functions $\phi: \mathscr{H} \times \mathbb{C} \rightarrow \mathbb{C}$ by

$$
\begin{aligned}
& \left.\phi\right|_{k+1 / 2, m}[\tilde{\alpha}, X, s]= \\
& \quad s^{m} \phi(\tau)^{-2 k-1} e^{m}\left(\frac{-c(z+\lambda \tau+\mu)^{2}}{c \tau+d}+2 \lambda^{2} \tau+2 \lambda z+\lambda \mu\right) \phi\left(\frac{a \tau+b}{c \tau+d}, \frac{z+\lambda \tau+\mu}{c \tau+d}\right),
\end{aligned}
$$

where $[\tilde{\alpha}, X, s] \in G^{J}$.

The Jacobi group for $\Gamma_{0}(4 N)$ is a subgroup $\Gamma_{0}^{J}(4 N)^{*}$ of $G^{J}$, given by

$$
\Gamma_{0}^{J}(4 N)^{*}=\left\{\left[\alpha^{*}, X\right]: \alpha \in \Gamma_{0}(4 N), X \in \mathbb{Z}^{2}\right\} .
$$

A Jacobi form $\phi(\tau, z)$ of weight $k+\frac{1}{2}$ and index $m$ for the group $\Gamma_{0}(4 M)$, with character $\chi$, is a holomorphic function $\phi: \mathscr{H} \times \mathbb{C} \rightarrow \mathbb{C}$ satisfying the following conditions:

(i) $\left.\phi\right|_{k+1 / 2, m}\left[\gamma^{*}, X\right](\tau, z)=\chi(d) \phi(\tau, z)$, where $\chi$ is a Dirichlet character mod $4 M$ and $\gamma=\left(\begin{array}{ll}a & b \\ c & d\end{array}\right) \in \Gamma_{0}(4 M)$.

(ii) For every $\alpha=\left(\begin{array}{ll}a & b \\ c & d\end{array}\right) \in \mathrm{SL}_{2}(\mathbb{Z})$, the image $\left.\phi\right|_{k+1 / 2, m}[\tilde{\alpha},(0,0)](\tau, z)$ has a Fourier development of the form

$$
\sum_{\substack{n, r \in \mathbb{Q} \\ r^{2} \leq 4 n m}} c_{\alpha}(n, r) e(n \tau+r z),
$$

where the sum ranges over rational numbers $n, r$ with bounded denominators subject to the condition $r^{2} \leq 4 n m$.

Further, if $r^{2}<4 n m$ whenever $c_{\alpha}(n, r) \neq 0$, then $\phi$ is called a Jacobi cusp form. We denote by $J_{k+1 / 2, m}(4 M, \chi)$ the space of Jacobi forms of weight $k+\frac{1}{2}$, index $m$ for $\Gamma_{0}(4 M)$ with character $\chi$, and by $J_{k+1 / 2, m}^{\text {cusp }}(4 M, \chi)$ the subspace of $J_{k+1 / 2, m}(4 M, \chi)$ consisting of Jacobi cusp forms. A Jacobi form $\phi$ has a Fourier expansion of the form

$$
\phi(\tau, z)=\sum_{\substack{n, r \in \mathbb{Z} \\ r^{2} \leq 4 n m}} c(n, r) e(n \tau+r z) .
$$

Since $c(n, r)=c\left(n^{\prime}, r^{\prime}\right)$ if $r^{\prime 2}-4 n^{\prime} m=r^{2}-4 n m$ and $r^{\prime} \equiv r(\bmod 2 m)$, we write the Fourier expansion of $\phi$ as

$$
\phi(\tau, z)=\sum_{\substack{0 \geq D, r \in \mathbb{Z} \\ D \equiv r^{2}(4 m)}} c_{\phi}(D, r) e\left(\frac{r^{2}-D}{4 m} \tau+r z\right) .
$$


Let $D<0$ be a discriminant and $r$ an integer modulo $2 m$ with $D \equiv r^{2}(4 m)$. Then the $(D, r)$-th Poincaré series, denoted by $P_{(D, r)}$, is defined by

$$
P_{(D, r)}(\tau, z)=\left.\sum_{\gamma \in \Gamma_{0}(4 M)_{\infty}^{J} \backslash \Gamma_{0}(4 M)^{J}} \bar{\chi}(\gamma) e(n \tau+r z)\right|_{k+1 / 2, m} \gamma
$$

We state the following proposition without proof.

Proposition 2.1. The Poincaré series $P_{(D, r)}$ lies in $J_{k+1 / 2, m}^{\mathrm{cusp}}(4 M, \chi)$ and satisfies

$$
\left\langle\phi, P_{(D, r)}\right\rangle=\alpha_{k, m}|D|^{-k+1} c_{\phi}(D, r),
$$

for each $\phi \in J_{k+1 / 2, m}^{\text {cusp }}(4 M, \chi)$, where $\alpha_{k, m}=\Gamma(k-1) m^{k-3 / 2} /\left(2 \pi^{k-1}\right)$. It has a Fourier development of the form

$$
P_{(D, r)}(\tau, z)=\sum_{\substack{0>D^{\prime}, r^{\prime} \in \mathbb{Z} \\ D^{\prime} \equiv r^{\prime 2}(4 m)}}\left(g_{D, r}\left(D^{\prime}, r^{\prime}\right)+\chi(-1) g_{D, r}\left(D^{\prime},-r^{\prime}\right)\right) e\left(\frac{r^{\prime 2}-D^{\prime}}{4 m} \tau+r^{\prime} z\right),
$$

where $D=r^{2}-4 m n, D^{\prime}=r^{\prime 2}-4 m n^{\prime}$, and $g_{D, r}\left(D^{\prime}, r^{\prime}\right)$ is given by

$$
\delta_{m}\left(D, r, D^{\prime}, r^{\prime}\right)+i^{-k-3 / 2} \pi \sqrt{\frac{2}{m}}\left(\frac{D^{\prime}}{D}\right)^{k / 2} \sum_{\substack{c \geq 1 \\ 4 M \mid c}} H_{m, c, \chi}\left(D, r, D^{\prime}, r^{\prime}\right) J_{k}\left(\frac{\pi \sqrt{D D^{\prime}}}{m c}\right),
$$

with

$$
\delta_{m}\left(D, r, D^{\prime}, r^{\prime}\right)= \begin{cases}1 & \text { if } D^{\prime}=D \text { and } r^{\prime} \equiv r(\bmod 2 m) \\ 0 & \text { otherwise }\end{cases}
$$

and

$$
\begin{aligned}
H_{m, c, \chi}\left(D, r, D^{\prime}, r^{\prime}\right)=c^{-3 / 2} e^{-r r^{\prime} /(2 m c)} & \\
& \times \sum_{\substack{d, \lambda(c) \\
d d^{-1} \equiv 1(c)}} \bar{\chi}(d)\left(\frac{c}{d}\right)\left(\frac{-4}{d}\right)^{1 / 2} e_{c}\left(d^{-1}\left(m \lambda^{2}+r \lambda+n\right)+d n^{\prime}-\lambda r^{\prime}\right) .
\end{aligned}
$$

\section{The Eichler-Zagier map}

First we consider the space $J_{k+1 / 2,1}^{\text {cusp }}(4 M, \chi)$. Put $D=D_{0} \ell^{2}, r=r_{0} \ell$ in Proposition 2.1. In the Fourier coefficient of $P_{\left(D_{0} \ell^{2}, r_{0} \ell\right)}$, the Kloosterman-type sum is periodic as a function of $\ell$ of period $2 c$. Hence, for any $h(\bmod 2 c)$, its Fourier transform (after replacing $\ell$ by $\ell d$ and $\lambda$ by $\lambda d$ ) becomes

$$
\begin{aligned}
\frac{1}{2 c^{5 / 2}} \sum_{\substack{\ell(2 c), d(c)^{*} \\
\lambda(c)}} \bar{\chi}(d)\left(\frac{c}{d}\right) & \left(\frac{-4}{d}\right)^{1 / 2} \\
& \times e_{2 c}\left(d\left(2 \lambda^{2}+2 r_{0} \ell \lambda+2 n_{0} \ell^{2}+2 n-2 r \lambda-r_{0} \ell r-h \ell\right)\right) .
\end{aligned}
$$


Since $4 \mid c$, the sum over $\lambda$ is nonzero only if $r_{0} \ell \equiv r(\bmod 2)$. Hence, the sum over $\lambda$ becomes

$$
\sum_{\lambda(c)} e_{c}\left(d \lambda^{2}\right) e_{c}\left(-d\left(\frac{r_{0} \ell-r}{2}\right)^{2}\right) .
$$

Again, the fact that $4 \mid c$ and $\operatorname{gcd}(c, d)=1$ gives the identity

$$
\frac{1}{\sqrt{2 i c}} \sum_{\lambda(c)} e_{c}\left(d \lambda^{2}\right)=\left(\frac{c}{d}\right)\left(\frac{-4}{d}\right)^{-1 / 2} .
$$

Thus, the Fourier transform simplifies to

$$
\begin{aligned}
\frac{\sqrt{i}}{\sqrt{2} c^{2}} \sum_{\ell(2 c), d(c)^{*}} \bar{\chi}(d) e_{4 c}\left(d \left(D_{0} \ell^{2}\right.\right. & +D-2 h \ell)) \\
& =\frac{\sqrt{i}}{4 \sqrt{2} c^{2}} \sum_{\ell(2 c), d(4 c)^{*}} \bar{\chi}(d) e_{4 c}\left(d\left(D_{0} \ell^{2}+D-2 h \ell\right)\right),
\end{aligned}
$$

which is the Fourier transform of the corresponding Kloosterman sum of integral weight.

More precisely:

Theorem 3.1. The Eichler-Zagier map $\mathscr{L}_{1}$ maps $J_{k+1 / 2,1}^{\text {cusp }}(4 M, \chi)$ into $S_{k}^{\square}(16 M, \chi)$. Proof. We shall prove that the $(D, r)$-th Fourier coefficient of $P_{\left(D_{0} \ell^{2}, r_{0} \ell\right)}$ is equal (up to constant) $|D|$-th Fourier coefficient of $P_{\left|D_{0}\right| \ell^{2}}$. It is easy to see that

$$
\delta_{1}\left(D_{0} \ell^{2}, r_{0} \ell, D, r\right)=\delta_{\left|D_{0}\right| \ell^{2},|D|} .
$$

We consider both the Kloosterman sums as periodic functions of period $2 c$. The arguments put forth above shows that for each $c \geq 1$, with $4 M \mid c$, the Fourier transform of $H_{1, c, \chi}\left(D_{0} \ell^{2}, r_{0} \ell, D, r\right)$ is equal to (up to the required constants) the Fourier transform of the Kloosterman sum (corresponding to integral weight) $K_{16 M, \chi}\left(\left|D_{0}\right| \ell^{2},|D| ; 4 c\right)$. This proves the theorem.

The index-changing operator $\boldsymbol{I}_{\boldsymbol{m}}$. If $\phi \in J_{k+1 / 2, m}^{\mathrm{cusp}}(4 M, \chi)$, define $I_{m}$ by

$$
\phi \mid I_{m}(\tau, z)=\sum_{\lambda(\bmod m)} e\left(\lambda^{2} \tau+2 \lambda z\right) \phi(m \tau, z+\lambda \tau) .
$$

Proposition 3.2. $I_{m}$ maps $J_{k+1 / 2, m}^{\text {cusp }}(4 M, \chi)$ into $J_{k+1 / 2,1}^{\text {cusp }}\left(4 m M, \chi \chi_{m}\right)$. The Fourier development of $\phi \mid I_{m}$ is of the form

$$
\phi \mid I_{m}(\tau, z)=\sum_{\substack{0<D, r \in \mathbb{Z} \\ D \equiv r^{2}(\bmod 4)}}\left(\sum_{\substack{s(\bmod 2 m) \\ s \equiv r(\bmod 2)}} c_{\phi}(D, s)\right) e\left(\frac{r^{2}-D}{4} \tau+r z\right) .
$$


Proof. It is easy to see that

$$
\phi\left|I_{m}(\tau, z)=m^{-k / 2-1 / 4} \sum_{\lambda(\bmod m)} \phi_{1 / \sqrt{m}}\right|_{k, 1}\left[\widetilde{\Delta}_{m},(\lambda, 0)\right](\tau, z),
$$

where $\phi_{1 / \sqrt{m}}(\tau, z)=\phi(\tau, z / \sqrt{m})$ and $\Delta_{m}$ is the diagonal matrix $\operatorname{diag}(\sqrt{m}, 1 / \sqrt{m})$. The proposition now follows directly from the preceding expression.

Using the equality $\mathscr{E}_{m}=I_{m} \mathscr{E}_{1}$, together with Theorem 3.1 and Proposition 3.2, we have:

Theorem 3.3. The map $\mathscr{E}_{m}$ takes $J_{k+1 / 2, m}^{\text {cusp }}(4 M, \chi)$ into $S_{k}^{\square}\left(16 m M, \chi \chi_{m}\right)$.

\section{Half-integral weight Jacobi forms of index 1 and index $p$}

In the case of integral weight Jacobi forms, the well-known map $V_{p}$ is a Heckeequivariant map from $J_{k, 1}$ into $J_{k, p}$ ( $p$ is a prime). If we replace $k$ by $k+\frac{1}{2}$, then we have a Hecke-equivariant map $V_{p^{2}}$ from $J_{k+1 / 2,1}(4 M)$ into $J_{k+1 / 2, p^{2}}(4 M)$, which was given by Tanigawa [1986]. Therefore, existence of a Hecke-equivariant map from index 1 into $p$ in the case of half-integral weight Jacobi forms seems to be a natural question.

As an application of the map $\mathscr{L}_{m}$, we show that there does not exist a Heckeequivariant map from $J_{k+1 / 2,1}^{\text {cusp }}(4)$ into $J_{k+1 / 2, p}^{\text {cusp }}(4)$.

Let

$$
N= \begin{cases}p & \text { if } p \equiv 1(\bmod 4) \\ p^{2} & \text { if } p \equiv 3(\bmod 4)\end{cases}
$$

Let $\psi(\bmod N)$ be a primitive Dirichlet character such that $\psi^{2}=\chi_{p}$. Let $R_{\psi}$ be the twisting operator defined as in (1). Then, $R_{\psi}$ maps $S_{k}\left(16 N^{2}, \chi_{p}\right)$ into $S_{k}\left(16 N^{2}\right)$ and commutes with Hecke operators $T_{n},(n, p)=1$. Further, if $f \in S_{k}\left(16 N^{2}, \chi_{p}\right)$, we have

$$
\left(f \mid R_{\psi}\right)\left|W_{p}=f\right| R_{\psi}
$$

where $W_{p}$ is the $W$-operator on $S_{k}\left(16 N^{2}\right)$ for the prime $p$.

Case 1: $p \equiv 3(\bmod 4)$. Let $f \in S_{k}\left(4 p, \chi_{p}\right)$ be a normalized Hecke eigenform. Since $f \mid R_{\psi} \in S_{k}\left(4 p^{4}\right)$ and it is an eigenform for all the Hecke operators and the $W$ operators, it is a newform in $S_{k}^{\text {new }}\left(4 p^{4}\right)$. Hence, by the theory of newforms, it is not equivalent to a level-1 Hecke eigenform.

Case 2: $\boldsymbol{p} \equiv \mathbf{1}(\bmod 4)$. Let $f \in S_{k}^{\text {new }}\left(4 p, \chi_{p}\right)$ be a normalized Hecke eigenform. Then, $f \mid R_{\psi} \in S_{k}^{\text {new }}\left(4 p^{2}\right)$. Since $f \mid R_{\bar{\psi}} \in S_{k}^{\text {new }}\left(4 p^{2}\right)$, and $\psi^{3}=\bar{\psi}$ (as $\psi^{2}$ is quadratic), we get $f \mid R_{\psi}$ and $f\left|R_{\psi}\right| R_{\chi_{p}}$ are newforms in $S_{k}^{\text {new }}\left(4 p^{2}\right)$. Thus, the form $f$ is not equivalent to a level-1 Hecke eigenform. Now, we let $f \in S_{k}\left(p, \chi_{p}\right)$. 
Arguments as above again show that $f$ is not equivalent to a level-1 Hecke eigenform.

Thus, we conclude that a normalized Hecke eigenform in $S_{k}\left(4 p, \chi_{p}\right)$ is not equivalent to a normalized Hecke eigenform in $S_{k}(4)$. In view of the mapping property proved in Theorem 3.3, we have proved:

Theorem 4.1. There is no Hecke-equivariant map from the space $J_{k+1 / 2,1}^{\text {cusp }}(4)$ into the space $J_{k+1 / 2, p}^{\text {cusp }}(4)$.

In this connection the following question seems natural.

What contribution do half-integral weight Jacobi forms of square-free index make to the construction of a "Maass space" (if one exists) for degree-2 Siegel modular forms of half-integral weight?

\section{References}

[Tanigawa 1986] Y. Tanigawa, "Modular descent of Siegel modular forms of half integral weight and an analogy of the Maass relation", Nagoya Math. J. 102 (1986), 51-77. MR 87i:11066 Zbl 0564.10031

Received November 1, 2004.

M. MANICKAM

mmmanickam@yahoo.com

DEPARTMENT OF MATHEMATICS

RKM VIVEKANANDA COLLEGE

MYLAPORE, CHENNAI 600004

INDIA

B. RAMAKRISHNAN

ramki@mri.ernet.in

HARISH-CHANDRA RESEARCH INSTITUTE

CHHATNAG ROAD

JHUNSI, ALlaHABAD 211019

INDIA 